Open Access

\title{
From shopping to eating: food safety practices among Chinese immigrants in Canada
}

\author{
Lichun Willa Liu
}

\begin{tabular}{l}
\hline Correspondence: \\
willaliu16@gmail.com \\
Centre for Women Studies in \\
Education, Ontario Institute for \\
Studies in Education, University of \\
Toronto, Toronto, ON M5S 1V6, \\
Canada
\end{tabular}

Correspondence: willaliu16@gmail.com Education, Ontario Institute for Studies in Education, University of Canada

\begin{abstract}
This section explores the changes in food safety practices among recent Chinese immigrants in Canada. Funded by the Public Health Agency of Canada (PHAC) (2009-2010), this section focuses on food safety practices in grocery shopping, food preparation/ cooking, food storage, and eating in restaurants. Based on data from a small-scale survey and two focus groups with recent Chinese immigrants in the Greater Toronto Area, as well as individual interviews with a community worker and two public health professionals, this section highlights the challenges and educational needs of newcomers along with the knowledge gaps among the newcomers and public health professionals, community health educators and workers.
\end{abstract}

Keywords: Food safety, Chinese immigrants, Food practices, Chinese Canadians

\section{Background}

Food safety is defined as the degree of confidence that food will not cause sickness or harm to people when it is prepared, served and eaten according to its intended use (FAO/WHO 2003).

Food can be mishandled at any number of stages during food preparation, handling and storage.

Contaminated raw foods, inadequate cooking, and consumption of food from an unsafe source were the factors most commonly associated with reported outbreaks of food-borne illness in homes (Medeiros et al. 2001). Food contamination creates an enormous social and economic burden on communities and their health system. It is estimated that in the last 20 years these economic losses amounted to nearly 1.0 to 1.2 billion dollars (Korel et al. 2003; Jevsnik et al. 2008). Study shows that between 50\% and $87 \%$ of reported food-borne disease outbreaks have been associated with the home (Redmond and Griffith 2003).

Despite the importance of food safety to our health and economy, studies show that people have inadequate knowledge about measures needed to prevent food-borne illness in the home (Medeiros et al. 2001). Common mistakes identified include serving contaminated raw food, cooking/heating food inadequately, having infected persons handle implicated food and practice poor hygiene (Unusan 2007). Continued efforts 
are required to educate people on the hazards of improper food handling (Finch and Daniel 2005; Li-Cohen and Bruhn 2002; Mitakakis et al. 2004).

Food safety has been a top priority of the Canadian government. However, research on food safety practice in Canadian homes is lacking. A systematic literature review indicates that, of a total of 323 international publications related to consumer food safety knowledge, perceptions and food safety behaviours, only 26 were Canadian studies, which occurred between 1998 and 2011. The majority of the Canadian studies $(n=22)$ were classified as non-peer-reviewed public opinion reports (POR) and only four were peer-reviewed publications, of which three publications were based on one research project (Nesbitt et al. 2014).

For many immigrants, food is an essential component of culture and identity that they carry from their home country to the country of destination (Koc and Welsh 2002). Chinese food and approaches to eating are important parts of Chinese culture and are central to the Chinese way of life. Influenced by the yin-yang principle, an important concept in traditional Chinese medicine, Chinese food emphasizes a balance of both fan (e.g. rice, noodles, and steamed buns, dumplings) and ts'ai (e.g. stir-fried vegetable and meat dishes) (Chang 1977), a proper combination of "hot" and "cold" food. Unlike everyday thinking in the West, where foods are commonly organized according to principles of "good" and "bad" (Lupton and Chapman 1995), there is no "good" or "bad" food but only "right" or "wrong" food in the Chinese ideas and beliefs about food. If imbalanced, or improperly combined, food may cause illnesses. If carefully selected and properly administered, food may be used to strengthen health or cure diseases: "Food, therefore, is also medicine" (Chang 1977: 10).

There are wide knowledge gaps in the area of traditional foods and food safety such as information on traditional foods, food handling and preparation practices, educational needs of public health officials and newcomers (Dietrich et al. 2007; Papadopoulos et al. 2009). There is an urgent need for public health organizations to address issues such as determining the diverse needs, challenges and barriers of different ethnic communities in understanding and accessing food safety-related information and public services.

So far most studies on food safety focus mainly on food productions, food processing and food transportation as well as food sold in supermarkets and stores, or served in restaurants. There is little research on food safety among different ethnic groups in Canada. Chinese immigrants make up one of the largest immigrant groups to Canada in the past decade (Citizenship and Immigration Canada 2009) and over half of them settled in the Greater Toronto Area. However, little is known about food safety knowledge and practices among Chinese immigrants. Only a limited number of studies have looked at food safety risks in common Chinese dishes (e.g. Chinese barbecued meats, Chinese fried rice) sold in many Chinese restaurants in North America (McElroy et al. 1999; Ying 2000).

This study explores the knowledge and practices relevant to food safety among Chinese immigrant newcomers in the Greater Toronto Area, Canada. In particular, we will look at what they learn and how they learn in adapting to the changes in grocery shopping, food preparation, cooking, and food storage in order to keep their food safe in their host country. We will also explore the knowledge gaps as well as the educational and informational needs of the Chinese newcomers. This study contributes to our knowledge and understanding of food safety and diversity in Canada, which is of 
crucial importance for public health professionals and community health educators in developing effective health educational programs for ethnic minority groups in Canada.

\section{Methods}

Multiple methods were used in data collection. Two focus groups and a small-scale survey were conducted with Chinese immigrants, who had been in Canada for 10 years or less and who were residing in the Greater Toronto Area at the time of participation in our research. Three individual interviews were conducted with front-line community workers serving the Chinese community in the GTA: a Community Nutrition Educator (CNE), a dietitian, and a health program coordinator at a community centre. A consultation meeting was conducted with Toronto Public Health officials and professionals.

The focus groups were conducted in Mandarin among two groups of women ( $n=6$ for both groups): middle-aged women (35-50 years of age) and older women (55 years or older), who reported primary responsibility for food work in the home. Consideration of age, marital status, number of children, level of education, years in Canada, as well as types of food work (e.g. cooking, and grocery shopping) were also taken into account when selecting participants for the focus groups. All the participants in the focus groups were asked to complete a survey questionnaire distributed at the end of the focus groups. Electronic copies of the survey questionnaire were sent to two email lists of Chinese immigrants in the GTA. Hard copies of the questionnaires were also distributed to recent Chinese immigrants at workshops and seminars.

Twenty-two valid copies of survey questionnaires were returned, including 12 hard copies and 10 electronic copies from both women (86\%) and men (14\%) ranging in age between 23 and 74. Most of the respondents were married (68\%), with at least one child in their family (59\%). Eighty two percent of the participants had an educational level of college/university and above. Ninety percent of the respondents were in Canada for less than 10 years, and $45 \%$ of them were newcomers, who had been in Canada for less than 3 years.

Two of the individual interviews were conducted in English and one in Mandarin, and the consultation meeting was conducted in English. A consultation meeting was conducted in English with public health professionals working with different ethnic communities in the GTA. All the interviews, focus groups and the consultation meeting lasted about 1-2 hours and were recorded and transcribed.

\section{Results}

\section{Changes in diet and food safety practices}

In our survey, the majority of respondents reported adopting new food items to their diet (91\%), such as bread (96\%), dairy products (91\%), pasta (82\%), and salads (71\%). Many of the respondents also reported increased consumption of meat (50\%), frozen food (55\%), dessert (55\%), and organic food (64\%) (see Fig. 1). Over two-thirds of the respondents reported maintaining a traditional diet (68\%), with rice and noodles as their staple foods, but slightly reduced consumption of steamed bread, another traditional Chinese staple food. However, in contrast to literature on Chinese immigrant dietary acculturation (Lv and Cason 2004; Satia 2000; Wang 2007), half of the respondents consumed less processed or ready-to-eat food than before. This is probably 


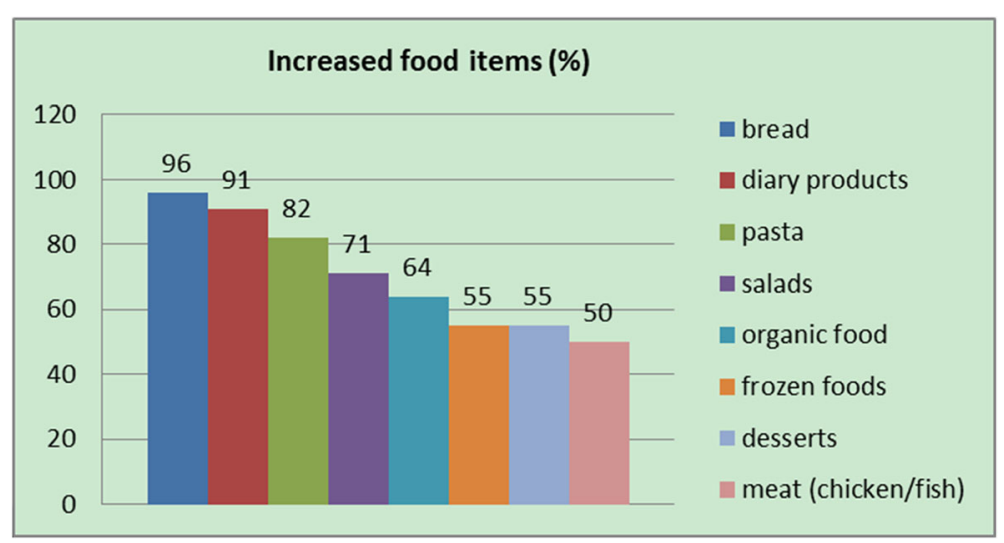

Fig. 1 Reported increased consumption of Non-Chinese food Items (\%)

because of their reduced source of income after immigration, as the majority of them (86\%) did not have a full-time job at the time of their participation in this research.

All the participants reported that they were concerned about food safety when they do grocery shopping. Many of them said that they would take the following factors into consideration when doing grocery shopping: expiry date (86\%), freshness (64\%), location of the stores (59\%), personal/family preferences (55\%), food prices $(50 \%)$, and nutrition (50\%). A relatively small number of the respondents said they cared about local food (41\%) or brand names of the food (36\%), which were likely to be more expensive and thus less affordable for the new immigrants.

The majority of people surveyed (82\%) said they were responsible for cooking in their family. While many respondents reported food safety as their top priority (73\%), the vast majority seemed to be more concerned about nutrition (96\%) and food preferences (86\%) when they cooked or prepared food for themselves and their family.

Over half of the respondents reported reduced frequency in eating out (55\%) since they immigrated to Canada. However, when they did eat out, food safety was reported as one of their top concerns (91\%), followed by cleanliness of the restaurants (77\%).

\section{Food safety-related learning}

Overall, nearly all the recent immigrants in the focus group felt that foods they eat are safer in Canada than in their home country, with reduced or strictly controlled use of pesticides, additives, and hormones. Compared to their home country, where food recall is often done only after reports of incidences of food-borne illness, the very recent newcomers felt that food safety alerts and food recalls were done as precaution to prevent diseases in Canada, well before the contaminated food reaches their table.

I feel that they have better food quality control here, and I don't see a lot of negative reports in the media on food safety. So I am less concerned about food safety now and normally don't check what I buy. (Min, 42, female, less than a year in Canada)

With the exception of one or two who said that they have always been concerned about food safety before and after immigration, the majority of the participants in the focus group said that they became more aware of food safety, nutrition and health since 
they came to Canada. Many also reported learning new practices in purchasing, preparing/cooking, and storing food. These include checking food labels for expiry date, using separate chopping boards for cutting raw meats and cooked food, and avoiding heating food in Styrofoam boxes. Many also reported changing their old habit of taking food with their own chopsticks by learning to use service spoons or chopsticks when serving meals and when eating from shared dishes. Several participants re-discovered some traditional ways of dish cleaning in order to reduce the use of detergents. These include using water from boiling noodles and dumplings in washing off grease from the dishes, and saving water from washing rice to help remove pesticide residues from fruits and vegetables. Many respondents found that these practices are effective, economical, and beneficial to their own health and the environment.

Ling is a woman in her mid-30 seconds and a mother of a 17-month-old daughter. Compared to other women in the focus groups, Ling is the most knowledgeable person in regards to food and health-a result of her long-time passion for food and eating. When she goes grocery shopping, Ling said that she checks not only the expiry date, but also the ingredients, such as the content of salt, fat, calcium, and vitamins, etc. When she cooks, Ling always uses separate chopping-boards and knives to cut meats and vegetables and assigns different bowls and containers for holding raw meats and cooked food. She also sanitizes all her cutleries after they are washed to keep them free from bacteria.

We don't use a dishwasher but use a sterilizer to sterilize dishes. I separate the dishes for raw meat and for cooked food. I also use different cloth to wash different things. For example, I use a white cloth to wash containers for raw food. (Ling, mid30 seconds, female)

Ling also reported learning to differentiate different types of plastic bags/containers when freezing or storing foods in the refrigerator. Whenever there was a food recall or an outbreak of diseases (e.g. SARS, mad-cow disease), Ling would search on-line to find more information on it and to check it out on the official public health websites both in Canada and the United States. In addition, Ling makes their own sausages at home, because she thinks homemade sausages are safer and healthier than those sold in the store.

\section{Sources of information on food safety}

Over half of participants said they learned something about food safety after immigration (55\%). The three major sources they used to acquire information on food safety were: TV (55\%), Internet (55\%), family and friends (50\%). Self-reported barriers to food safety in the survey include language barrier (22\%), not knowing where to find such information (27\%), and lack of time (32\%). Despite the low percentage of report on language barrier, the vast majority of the participants preferred to receive food safety information in their mother tongue (82\%), through public media (e.g. TV, newspapers/ books/magazine) (68\%), the internet (59\%), and from family and friends (50\%). Only a small number of participants reported getting food safety information from the government $(27 \%)$ or the community centre $(32 \%)$. In terms of their interest in food safetyrelated learning, two-thirds of the respondents reported learning about food-borne diseases $(68 \%)$, followed by learning on food storage (59\%) and food preparation (50\%). 


\section{Challenges in food safety practices}

In both focus groups with the Chinese immigrants and in the interviews with community workers and health educators, the English language was viewed as the biggest challenge for Chinese immigrants, especially newcomers and older adults, in accessing information on food safety. Here is a quote from Yue, a woman in her mid-40 seconds who had been in Canada for less than a year when she participated in this research:

I don't know how to get food safety information, as everything is in English. Even if it is available, I don't think I have the time to sit down and search for such information or attend workshops. Even if I decide to check it out, I think I will get it from the free Chinese language newspapers like $\mathrm{Du}$ shi Bao (Metropolitan News) and Beimei Shibao (North American Journals) or the Chinese-language website like www.51.ca for new immigrants from Mainland China.

As a brand-new immigrant without a full-time job, Yue said she is most concerned about food prices. "Every week I would get the flyers, and see what's on sale, and then run from store to store to get them." When asked if she has any concerns for the safety of those on-sale foods, Yue said, "I feel the food is so safe here that I am never worried about it, and never bothered to check it."

Juan is a 63-year-old woman who came to Canada 8 years ago. Despite her efforts in learning English through LINC/ESL classes and by watching TV and reading newspapers, Juan found her English not enough for her to understand food labels when grocery shopping, and to read information on food safety and disease prevention such as H1N1. "My English level is still relatively low. I always have to use a dictionary to understand the news and the pamphlets distributed by the government," Juan said.

Fang, a 61-year-old woman, came to Canada two years earlier to help her daughter with childcare. Fang talked about the challenges in adjusting to changes in her food work.

I didn't pay much attention to those things before. I used to think I had done well enough. But when I came here, there are suddenly so many things I need to pay attention to. The young people, they care about so many things. For example, after I cut the meat, I was required to sanitize the knives. I need to wash the chopping board with detergent, rinse it thoroughly with hot water, and then hang it to dry it....

\section{Gaps in food-safety practices}

The research on Chinese immigrants' food safety practices reveals several gaps in their food safety knowledge and practices. Many showed great concern for food safety (64\%) and expressed desire of learning more in the following aspects: food-borne diseases (68\%), food preparation and storage (59\% respectively), junk food $(50 \%)$ and how to grow their own food in an urban context (50\%) (See Fig. 2).

Furthermore, most of the participants lack adequate knowledge of Canadian food safety guidelines and recommended food handling practices. For example, many respondents reported washing hands before and after handling food, but they did not always use soap and water nor did they always wash for the recommended 20 seconds. Quite a number of the participants were not aware that it is not safe to defrost meat/ thawing frozen meat over the counter at room temperature. Almost none of the 


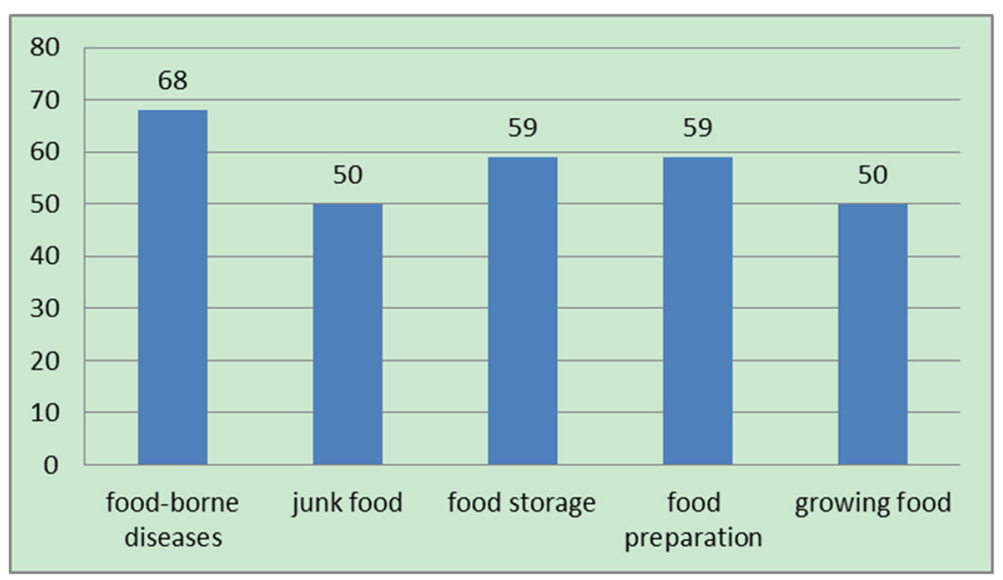

Fig. 2 Informational Needs (\%)

Chinese participants reported using a thermometer to test the temperature when cooking meat. When storing food, none of the participants were aware of the recommended time (within 2 hours of cooking) and temperature for refrigerating or freezing cooked food. Neither did any of the participants reported dating food before putting it in the refrigerator.

\section{Recommendations}

a. Recommendations from Chinese immigrants

- Holding lectures about food safety for people of all ages, especially the seniors.

- Hiring more Mandarin-speaking health promoters to deliver the information and translate the most important information about food safety in different ethnic languages.

- To maximize the benefits of the resources in a more economic way, public health organizations can have these workshops videotaped, put on their websites or shown at community centres where new immigrants can easily get access to them.

b. Recommendations from Public Health professionals:

- Hire more educators who speak ethnic language/seconds (i.e. Mandarin), to reach out to the new immigrants with language barriers.

- Translate some of the most important information on food safety into ethnic language/seconds (so far only the Canadian food guide has been translated into different languages). Distribute them not only through public health websites, but also through traditional means of pamphlets, community centres, schools, early child centres, doctors' offices, etc.

- Develop programs to reach out to isolated groups (either because of language, health problems, poverty); people with mental health issues; low-income families 
who use the community kitchen or food bank. Engage in more outreach because many new immigrants often do not know where to get the information.

- Develop/create effective ways to disseminate information on food safety, to make the learning more engaging, e.g. incorporate such knowledge into extra-curriculum activities, like community kitchens, etc.

- Recognize the need for educating newcomers about food allergies-peanut, seafood, etc., proper practices in defrosting meat, and in washing (as well as over washing) fruits and vegetables.

\section{Conclusions}

This chapter examined a small sample of recent Chinese immigrants in Toronto, their concerns on food safety and health, their informational/educational needs, as well as their recommendations for improving immigrant food safety awareness and practices. This chapter also looked at recommendations by local community nutritionists and public health workers serving immigrant communities. It is clear that we need to recognize the diversity among newcomers in terms of social and cultural capital, level of knowledge, and life experiences. Meanwhile, age, level of education, years of Canadian residency, degree of fluency in English and socio-economic status, clearly can create barriers beyond an assumed ethnic origin. Programs targeting newcomer immigrant communities need to have flexibility in addressing such diversity. This, of course, comes down to availability of public health service workers who are not only informed about these communities but can serve as frontline workers in the knowledge translation and transfer. Further research is needed to explore intergenerational knowledge gaps and food safety risks posed by them.

We should also recognize that knowledge transfer must be seen as a mutual exchange and not a one-way transmission. This implies recognizing and benefitting from the indigenous knowledge and best practices of diverse global cultures, while at the same time informing newcomers about current Canadian safety and nutrition standards. Partnerships between communities, food safety professionals, practitioners and academics can create mutual learning opportunities and facilitate exchange of knowledge and experience to bridge these gaps, resulting in an increased understanding of traditional foods and food handling practices.

\section{Acknowledgements}

This work was funded by the Public Health Agency of Canada. The author wishes to thank Mustafa Koc and Ozlem Guclu-Ustundag for their contributions to this project. The author would also like to give special thanks to the Chinese immigrants who participated in the study.

\section{Competing interests}

The author declares that he/she has no competing interests.

\section{Publisher's Note}

Springer Nature remains neutral with regard to jurisdictional claims in published maps and institutional affiliations. 
Dietrich, S., Isaacs, S., Marshall, B. J., \& Nesbitt, A. (2007). Final report: Food safety and traditional food. Division of Foodborne, Waterborne and Zoonotic Division. Ottawa: Public Health Agency of Canada.

Joint FAO/WHO Codex Alimentarius Commission, Joint FAO/WHO Food Standards Programme, \& World Health Organization. (2003). Codex Alimentarius: Food hygiene, basic texts. Food \& Agriculture Org.

Finch, C., and E. Daniel. 2005. Food safety knowledge and behavior of emergency food relief organization workers: Effects of food safety training intervention. Journal of Environmental Health 67 (9): 30.

Jevsnik, M., Hebec, V., \& Raspor, P. (2008). Consumers' awareness of food safety from shopping to eating. Food control, 19 (8): 737-745.

Koc, M., and J. Welsh. 2002. Food, identity and immigrant experience. Canadian Diversity 1 (1): 46-48.

Korel, F., B. Ergonul, and E. Gokgoz. 2003, 2003. Economic impacts of HACCP system applications in food industry. Food: 80-82.

Li-Cohen, A.E., and C.M. Bruhn. 2002. Safety of consumer handling of fresh produce from the time of purchase to the plate: A comprehensive consumer survey. Journal of Food Protection 65 (8): 1287-1296.

Lupton, D., and S. Chapman. 1995. 'A healthy lifestyle might be the death of you': Discourses on diet, cholesterol control and heart disease in the press and among the lay public. Sociology of Health and IIIness 17: 477-494.

LV, N., and K.L. Cason. 2004. Dietary pattern change and acculturation of Chinese Americans in Pennsylvania. Journal of American Diet Association 104 (5): 771-778.

McElroy, D.M., L. Jaykus, and P.M. Foegeding. 1999. A quantitative risk assessment for Bacillus Cereus emetic disease associated with the consumption for Chinese-style rice. Journal of Food Safety 19 (3): 209-229.

Medeiros, L., V. Hillers, P. Kendall, and A. Mason. 2001. Evaluation of food safety education for consumers. Journal of Nutrition Education 33: S27-S34.

Mitakakis, T.Z., M.I. Sinclair, C.K. Fairley, P.K. Lightbody, K. Leder, and M.E. Hellard. 2004. Food safety in family homes in Melbourne, Australia. Journal of Food Protection 67 (4): 818-822.

Nesbitt, A., M.K. Thomas, B. Marshall, K. Snedeker, K. Meleta, B. Watson, et al. 2014. Baseline for consumer food safety knowledge and behaviour in Canada. Food Control 38: 157-173.

Papadopoulos, A., S. Isaacs, and B. Marshall. 2009. Cultural diversity in food safety issues: Identifying challenges, knowledge gaps and opportunities for the future. Guelph: University of Guelph.

Redmond, E.C., and C.J. Griffith. 2003. Consumer food handling in the home: A review of food safety studies. Journal of Food Protection 66 (1): 130-161.

Satia, A. J. (2000). Diet, acculturation, and health in Chinese-American women.

Unusan, N. 2007. Consumer food safety knowledge and practices in the home in Turkey. Food Control 18 (1): 45-51.

Wang, C. (2007). From China to the United States: Nutrition, diet and acculturation of Chinese employed in high-tech industries. Results from a Web-based survey. University of Maryland, College Park.

Ying, J. 2000. Chinese-style barbecue meats: A public health challenge. Canadian Journal of Public Health 91 (5): 386-389.

Submit your manuscript to a SpringerOpen ${ }^{\circ}$ journal and benefit from:

- Convenient online submission

- Rigorous peer review

Open access: articles freely available online

High visibility within the field

Retaining the copyright to your article

Submit your next manuscript at $>$ springeropen.com 